\title{
STOCHASTIC RESONANCE IN SYSTEM WITH ON-OFF INTERMITTENCY
}

\author{
A. KraWIECKI \\ Institute of Physics, Warsaw University of Technology \\ Koszykowa 75, 00-662 Warsaw, Poland
}

(Received June 5, 1997; in final form September 18, 1997)

\begin{abstract}
Stochastic resonance in a chaotic threshold-crossing system exhibiting on-off intermittency and attractor bubbling: the logistic map with the control parameter varying randomly or chaotically in time is studied in the case of weak additive and multiplicative periodic forcing. In both cases signal-to-noise ratio shows dependence on the forcing frequency; in the case of multiplicative forcing this dependence appears even for very small frequencies. It is shown that this is a result of a very long characteristic time scale, typical of systems with on-off intermittency.
\end{abstract}

PACS numbers: $05.45 .+b, 05.40 .+j$

\section{Introduction}

The primary signature of stochastic resonance (SR) [1] is that addition of random (stochastic) noise can improve the signal-to-noise ratio (SNR) at the output of a periodically modulated nonlinear system (for review see e.g. [2, 3] and conference proceedings $[4,5])$. So far, the appearance of SR has been theoretically predicted e.g. in bistable [6] and monostable [7] systems and in both dynamical [8] and non-dynamical [9] threshold-crossing systems. The occurrence of SR in systems in which chaotic rather than stochastic dynamics was used to improve SNR was also reported e.g. in [10-12]. In the latter case mainly systems with some kind of bistability (e.g. Chua's circuit or one-dimensional maps in the vicinity of a boundary crisis) were investigated. Only recently SR in a system with Pomeau-Maneville type-III intermittency (spin-wave dynamics in nonlinear ferromagnetic resonance) was investigated experimentally and theoretically [13]. In this case the phases of periodic and chaotic motion play a role of the two states in a bistable system, and under the influence of external periodic forcing their sequence has a strong periodic component. Investigation of SR in chaotic systems is interesting because it offers a possibility to observe noise-free $\mathrm{SR}$ in which appropriate properties of internal dynamics of a system are used to amplify SNR instead of an external noise.

This paper deals with the problem of the influence of additive and multiplicative periodic forcing on a model system exhibiting on-off intermittency 
(OOI) [14-16] and attractor bubbling [17-19]. The investigation is based mainly on numerical simulations. It turns out that $\mathrm{SR}$ is observed as the system control parameter is increased. The dynamics of the system is such that SR appears naturally in the same way as in dynamical threshold-crossing systems [8]. The possibilities of experimental realization are also discussed.

\section{The model}

In the simplest version of the stochastic resonator proposed by Wiesenfeld et al. [8] a particle moving in a potential well of finite depth under the influence of stochastic force is considered. It spends most of the time near the bottom of the well but sometimes is thrown out of the well. Next, the particle is deterministically reinjected into the well after certain refractory time and a short pulse is produced at the resonator output. If the force acting on the particle has also a weak periodic component with frequency $f_{\mathrm{s}}$, delta-spikes located at the multiples of this frequency appear in the power spectral density (PSD) of the output pulse train and SNR as a function of the input noise strength exhibits a maximum. In this paper a chaotic threshold-crossing system is considered whose dynamics is very similar to the above-described one. Namely the logistic map with the control parameter randomly or chaotically varying in time and with a weak additive or multiplicative periodic forcing is investigated

$$
y_{n+1}=\left[a x_{n}+\varepsilon\left(1+\cos 2 \pi f_{\mathrm{s}} n\right)\right] y_{n}\left(1-y_{n}\right)+\delta\left(1+\cos 2 \pi f_{\mathrm{s}} n\right)+\xi x_{n}^{\prime} .
$$

In Eq. (1) $n$ is the time step number, $y_{n}$ is the dynamical variable under investigation, $0 \leq x_{n} \leq 1$ is a random or chaotic variable, $0 \leq a \leq 4$ is the control parameter, $\varepsilon$ and $\delta$ are amplitudes of the multiplicative and additive periodic forcing, and $x_{n}^{\prime}$ is another random variable independent of $x_{n}$ which represents additive thermal noise with amplitude $\xi\left(x_{n}^{\prime}\right.$ is assumed to be non-correlated random noise with uniform distribution at $[0,1)$ and $\xi \ll 1$ so that $y_{n}$ does not leave the interval $[0,1)$ ).

In the case $\varepsilon=\delta=0$ the system (1) is a generic model for OOI [15], a phenomenon occurring in systems with chaotic attractors contained inside invariant subspaces for certain control parameter values. If $\xi=0, x_{n}$ is a random non-correlated variable with uniform distribution at $[0,1)$ (this is the so-called class-II OOI case [20]) and $a<a_{\mathrm{c}}=\mathrm{e}=2.718 \ldots$ then $y_{n}$ always approaches the invariant subspace $y_{n}=0$, independently of the choice of initial conditions. Inside this surface there is a stable, noisy attractor $0 \leq x_{n}<1$. If $a>a_{\mathrm{c}}$ but still $a \approx a_{\mathrm{c}}$ the so-called blowout bifurcation [21] occurs and the system exhibits OOI: during long laminar phases $y_{n}$ remains practically equal to zero but occasionally increases rapidly and a chaotic burst appears. The probability that the laminar phase has length $\tau$ obeys a power-law scaling $P(\tau) \propto \tau^{-3 / 2}$ and the mean duration of the laminar phases decreases according to another power law $\langle\tau\rangle \propto\left(a-a_{\mathrm{c}}\right)^{-1}[15]$. For other choices of $x_{n}$, e.g. various chaotic time series, these scaling laws remain valid, only with different proportionality constants and different values of the OOI threshold $a_{\mathrm{c}}$. In particular, if $x_{n}$ is a chaotic variable with a multi-peaked invariant density we have class-I OOI [20] for which e.g. $\langle\tau\rangle$ decreases rapidly with the rise of $a$. Before the blowout bifurcation occurs the subspace $y_{n}=0$ loses the asymptotic stability for $a>a_{\mathrm{b}}=1$. Then under the influence of any small perturbation 
destroying the invariant subspace, e.g. thermal noise $\xi>0$, the bursts characteristic of OOI appear already for $a>a_{\mathrm{b}}$, below the OOI threshold. This phenomenon is called attractor bubbling [17].

For the occurrence of $\mathrm{OOI}$ the external variation of the control parameter in time is not necessary. The analysis of all systems with OOI may be carried out assuming that there exists an effective, chaotically time-dependent control parameter which governs the system dynamics in the direction perpendicular to the invariant subspace [14]. This parameter is, in general, a function of both the system control parameter (which is constant in time) and state variables. Thus the phenomenon of SR in systems with OOI may be considered as a kind of noise-free $\mathrm{SR}$, as in the Pomeau-Maneville type-III intermittency [13].

\section{Results}

Let us now turn to the results of the numerical experiment with Eq. (1) for various $\varepsilon>0$ or $\delta>0$ and $f_{\mathrm{s}}$. Since the system has a parameter-controlled time scale (the mean duration of the laminar phase) the occurrence of SR with varying $a$ may be expected. The numerical tests reveal that this is really the case. Instead of $y_{n}$ a two-state approximation $Y_{n}$ of the full signal was analyzed: $Y_{n}=\Theta\left(y_{n}-y_{\mathrm{thr}}\right)$, where $\Theta(\cdot)$ is the Heaviside unit step function and $y_{\mathrm{thr}}$ was an arbitrarily chosen threshold for a burst. Typically $y_{\mathrm{thr}}=0.01$ was assumed; it is known that assuming such threshold value enables one to distinguish well between laminar phases and bursts in Eq. (1) without any periodic forcing, i.e. this yields scaling laws for laminar phases in accordance with theoretical predictions [15]. Thus the time series consisted of a sequence of pulses of unit height and various lengths rather than of short pulses with equal lengths as in $[8,9]$. A similar encoding was used in a simple threshold-crossing non-dynamical detector in [22], but in that paper the distribution of the pulse lengths was determined mainly by the correlation time of the noise while here it is determined by the dynamics of the system (1). Both for additive and multiplicative periodic forcing peaks in the PSD $S(f)$ at odd and even multiples of $f_{\mathrm{s}}$ were seen. SNR at the $n$-th harmonic of $f_{\mathrm{s}}$ $(n=1,2,3)$ was evaluated as SNR $=10 \log \left[S_{\mathrm{P}}\left(n f_{\mathrm{s}}\right) / S_{\mathrm{N}}\left(n f_{\mathrm{s}}\right)\right]$. Here $S_{\mathrm{N}}\left(n f_{\mathrm{s}}\right)$ is the PSD of the noise background at $n f_{\mathrm{s}}$ and $S_{\mathrm{P}}\left(n f_{\mathrm{s}}\right)=S\left(n f_{\mathrm{s}}\right)-S_{\mathrm{N}}\left(n f_{\mathrm{s}}\right)$ is the respective peak height. As SNR measured in this way is sensitive to the bandwidth $\Delta f$ all measurements were normalized to a standard bandwidth $\Delta f=2^{-12}$ [6].

Additive periodic forcing. If $\varepsilon=0$ and $\xi=0$ then setting $0<\delta \ll y_{\mathrm{thr}} / 2$ is equivalent to switching on a periodic perturbation transverse to the invariant subspace $y_{n} \doteq 0$ and for $a>a_{\mathrm{b}}=1$ the noisy attractor contained inside this subspace exhibits bubbling. First, the case when $x_{n}$ is a random non-correlated variable with uniform distribution at $[0,1)$ (class-II OOI) will be analyzed. Examples of the SNR vs. $a$ curves at three first harmonics of $f_{\mathrm{s}}$ are depicted in Fig. 1a-c. The shape of the SNR curve measured at the first harmonic is characteristic of SR. In Fig. 1a, which shows results for $\delta=4 \times 10^{-4}$, the maximum of the SNR is located above $a=a_{\mathrm{b}}$ but below $a=a_{\mathrm{c}}$ for a wide range of frequencies $f_{\mathrm{s}}$, thus here SR in attractor bubbling is obtained. It should be emphasized that $y_{n}$ during the bursts can exceed $y_{\text {thr }}$ by an order of magnitude (Fig. 1d), thus the dynamics of the system (1) plays an important role in the occurrence of SR in this case. The 


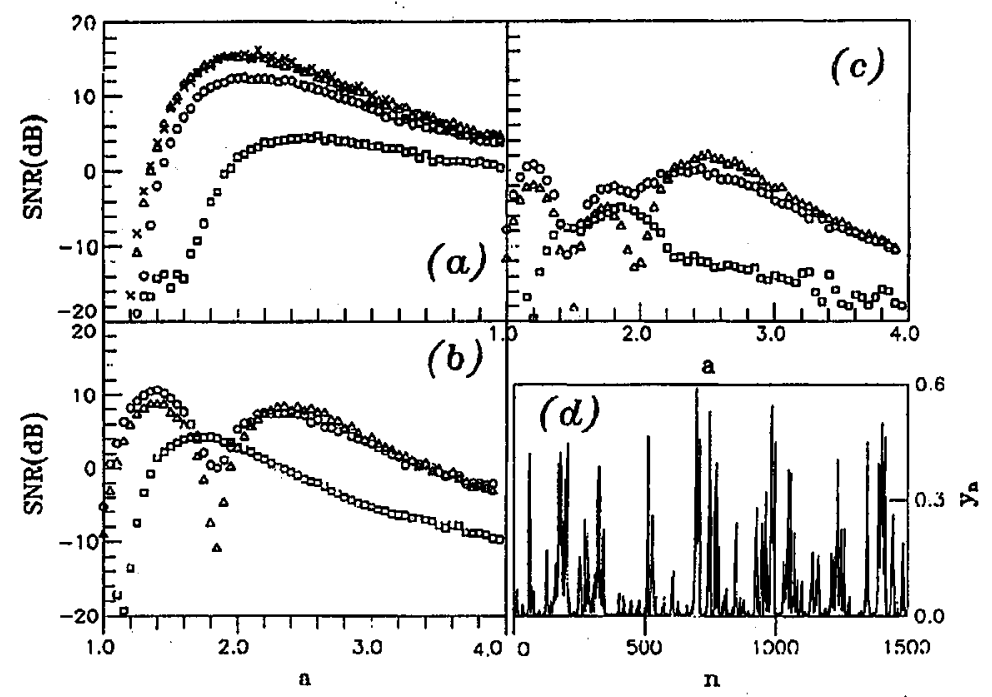

Fig. 1. SR in the map (1) with additive periodic forcing (class-II OOI). (a) SNR at the first harmonic, $\delta=4 \times 10^{-4}, f_{s}=1 / 8(\square), 1 / 16(0), 1 / 32(\Delta), 1 / 128(\times)$; (b,c) SNR at the second and third harmonics, respectively, $\delta=1.0 \times 10^{-3}, f_{\mathrm{s}}=1 / 8(\square), 1 / 128(0)$, $1 / 512(\triangle)$; (d) time series for $y_{n}$ at $a=2.5, \delta=4 \times 10^{-4}, f_{\mathrm{s}}=1 / 8$.

maximum value of SNR and its location strongly depend on the frequency of periodic forcing for high $f_{\mathrm{s}}$, an effect rather characteristic of SR in bistable systems, but saturate for low frequencies. For $f_{\mathrm{s}}=2^{-3}$ a small additional peak occurs at $a \approx 1.4$ (hardly visible) which disappears for decreasing $f_{\mathrm{s}}$, an effect also typical of bistable systems [23]. SNR at higher harmonics also exhibits SR (Fig. 1b, c). As the forcing frequency decreases, SNR at the second and third harmonics of the forcing frequency increases and in both cases exhibits characteristic dips called noise-induced resonances (Fig. 1b, c) [24].

If the amplitude of additive forcing $\delta$ is increased, SNR also increases and its maximum is shifted towards smaller values of $a$. The dependence of the SNR on the forcing frequency, seen in Fig. 1a, decreases. Addition of a certain amount of thermal noise $\xi \neq 0$ has a similar effect. If $\delta \approx y_{\mathrm{thr}} / 2$ but still $\delta<y_{\mathrm{thr}} / 2$ and $\xi=0$ or if $\delta / 2+\xi \approx y_{\text {thr }}$ the maximum of the SNR is observed at very small $a$, below the bubbling threshold $a_{\mathrm{b}}$. In this regime the mechanism for the occurrence of $\mathrm{SR}$ is the same as in the case of non-dynamical threshold-crossing detectors [9]: The dynamics of $y_{n}$ is mainly governed by the two last terms on the right hand side of Eq. (1) with the first term being a source of small additional noise with non-uniform distribution (cf. [18]). SNR in this limit exceeds that obtained in the dynamical regime discussed previously. This limit is not interesting from the point of view of chaotic dynamics.

As an example of SR in a system exhibiting class-I OOI, Eq. (1) with $x_{n}$ generated by another logistic map $x_{n+1}=r x_{n}\left(1-x_{n}\right)$ with $r=3.75$ is considered. For this value of $r$ the logistic map has a multi-peaked invariant density and thus 

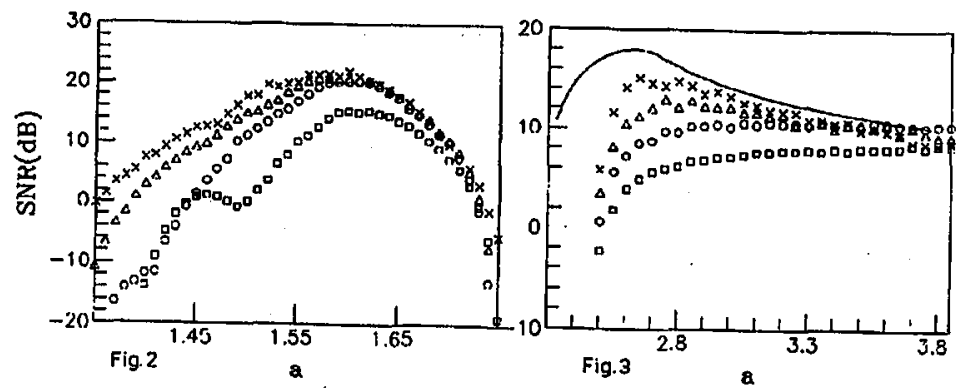

Fig. 2. SR in the map (1) with additive periodic forcing (class-I OOI). SNR at the first harmonic, $\delta=4 \times 10^{-4}, f_{s}=1 / 8(\square), 1 / 16(o), 1 / 32(\Delta), 1 / 128(\times)$.

Fig. 3. SR in the map (1) with multiplicative periodic forcing. SNR at the first harmonic, $\varepsilon=0.05, f_{s}=1 / 8(\square), 1 / 32(0), 1 / 512(\triangle), 1 / 2048(\times)$, solid line - result of the simplified adiabatic approximation (see text).

the value of $\langle\tau\rangle$ increases rapidly as $a$ exceeds $a_{\mathrm{c}}=1.673 \ldots$ [15]. The SNR at the first harmonic of the forcing frequency is shown in Fig. 2 for $\delta=4 \times 10^{-4}$ and $\xi=0$ and again SR in attractor bubbling (for $\dot{a}<a_{\text {c }}$ ) can be seen. The properties of SR in this case are very similar to the ones in the case of a system with class-I OOI. However, the width of the SR peak is small when compared to the result in Fig. 1a and SNR decreases rapidly with $a$ after passing through the maximum. In the class-I OOI case it is extremely difficult for $y_{n}$ to approach the invariant surface $y_{n}=0$ [20] and this must be true also in the regime of attractor bubbling, so $Y_{n} \equiv 1$ if $a \gg a_{\mathrm{b}}$ and the periodicity of the perturbing term is unimportant in comparison with its repulsive action in the vicinity of the invariant surface $y_{n}=0$. Another interesting property is that the maximum SNR exceeds in this case the one produced by $x_{n}$ taken as random non-correlated variable.

Multiplicative periodic forcing. The SNR curves obtained for $\delta=\xi=0$, $\varepsilon=0.05$ and with $x_{n}$ being a random non-correlated variable with uniform distribution at $[0,1)$ are shown in Fig. 3 for decreasing frequencies $f_{\mathrm{s}}$. If $f_{\mathrm{s}}=0.125$ only monotonic increase in SNR with $a$ is observed within the borders allowed for $a$ : $0<a<4-2 \varepsilon$. The maxima in SNR vs. $a$ curves can be seen only for low frequencies of the periodic forcing. Their values increase and their location shifts towards smaller values of $a$ as $f_{\mathrm{s}}$ decreases. SNR does not saturate even for very small frequencies of the multiplicative periodic forcing. This result may be compared with the predictions of the following simplified adiabatic approximation $[6,8]$.

In the adiabatic limit, using the continuous time approximation, the threshold crossing rate $\alpha(t)$ for the system (1) depends periodically on time, as under the influence of the periodic multiplicative forcing also the OOI threshold $a_{\mathrm{c}}$ becomes periodically time dependent. The instant value of $a_{\mathrm{c}}(t)$ for given $t$ may be obtained from the solution of the equation [15]

$$
F\left(a_{c}(t), p(t)\right)=0, \quad F(a, p)=a^{-1}[(p+a) \ln (p+a)-a-p \ln p],
$$

where $p(t)=\varepsilon\left(1+\cos 2 \pi f_{\mathrm{s}} t\right)$ is the multiplicative periodic forcing. For $\varepsilon=0$ and $a$ just above $a_{c}=$ e the mean laminar phase length scales as $\langle\tau\rangle=\mathrm{e}^{2} /\left[2\left(a-a_{\mathrm{c}}\right)\right][15]$. Just above the OOI threshold the bursts are rare and short, therefore neglecting 
their influence on the threshold crossing rate yields $\alpha=2\left(a-a_{\mathrm{c}}\right) / \mathrm{e}^{2}$, for $a>a_{\mathrm{c}}$. Thus for $0<\varepsilon \ll 1$ one gets for $\alpha(t)$

$$
\alpha(t)= \begin{cases}0 & \text { if } t \text { is such that } a<a_{\mathrm{c}}(t), \\ 2\left[a-a_{\mathrm{c}}(t)\right] / \mathrm{e}^{2} & \text { if } t \text { is such that } a>a_{\mathrm{c}}(t) .\end{cases}
$$

Expanding the rate (3) in the Fourier cosine series at $\left[0, T_{\mathrm{s}}\right)$, where $T_{\mathrm{s}}=1 / \mathrm{f}_{\mathrm{s}}$ is the forcing period, and utilizing the results of [8] yields the expression for SNR at the first harmonic: SNR $=10 \log \left[\alpha_{1}^{2} /\left(4 \alpha_{0} \Delta f\right)\right]$, where $\alpha_{n}$ is the $n$-th Fourier coefficient and the dependence on the bandwidth $\Delta f$ was included. This approximation for SNR is evidently wrong if the bursts occur frequently and their a verage length is comparable to the one of laminar phases, but just above the onset of OOI this prediction should be at least qualitatively correct. Surprisingly, as shown in Fig. 3, this is not the case. The numerical value of SNR is by some $\mathrm{dB}$ smaller than the one evaluated on the basis of the above approximation, and, what is more important, SNR approaches zero for $a$ considerably greater than it may be expected from the simple theory. It turns out that $y_{n}$ falls to zero if $a<2.47$ for the range of frequencies investigated in Fig. 3 , and no bursts appear for such $a$, so in the two-state approximation SNR $=0$.

The latter discrepancy is rather not a result of the approximations applied but has its roots in the "averaging" properties of systems with OOI. The time-independent threshold for OOI with multiplicative periodic forcing $\tilde{a}_{\mathrm{c}}$ may be evaluated as in the case $\varepsilon=0$, by requiring that the time-averaged Lyapunov exponent in the direction perpendicular to the invariant surface $y_{n}=0$ was equal to 1 [15]. This yields the condition

$$
\left\langle\ln \left[\tilde{a}_{\mathrm{c}} x_{n}+\varepsilon\left(1+\cos 2 \pi f_{\mathrm{s}} n\right)\right]\right\rangle=T_{\mathrm{s}}{ }^{-1} \sum_{n=0}^{T_{\mathrm{s}}-1} F\left(\tilde{a_{\mathrm{c}}}, p(n)\right)=0,
$$

where $\langle\cdot\rangle$ denotes the average over one period $T_{\mathbf{s}}$. For example, if $x_{n}$ is a random variable with uniform distribution and $T_{\mathrm{s}}=8$, this yields $a_{\mathrm{c}}=2.477$, in agreement with the numerical results. Therefore the system (1) with $\varepsilon>0$ possesses a well-defined, time-independent OOI threshold. It seems that even for small $f_{\mathrm{s}}$ this "averaging" tendency prevails and only in the limit of extremely small $f_{\mathrm{s}}$ the OOI threshold becomes time dependent and closely follows the periodic forcing term; the characteristic time scale of the system is simply very long. Thus the theoretical description of SR in this case must go beyond the adiabatic approximation even for small $f_{\mathrm{s}}$ and it is still an open problem.

\section{Discussion and conclusions}

In the present paper, the effect of additive or multiplicative periodic forcing on a model of chaotic threshold-crossing system exhibiting OOI and attractor bubbling was investigated. In both cases SR was obtained in the two-state approximation. In the case of additive forcing SNR depends on the forcing frequency, but for small $f_{\mathrm{s}}$ it is frequency independent. In this case many properties of SR are similar to the ones obtained when this effect is investigated in bistable systems. This probably may be explained by the fact that intermittent bursts are not single 
spikes, but their lengths have a certain distribution. Thus the two-state approximation produces a signal similar to what can be expected in asymmetric bistable systems. A close connection between such systems and threshold-crossing dynamics was pointed out in [25]; moreover, a dynamical system, but without OOI a noise-driven semiconductor diode which produces time series consisting of short pulses of light of various length was proposed to look for SR in [26]. In the case of multiplicative forcing SNR continues increasing even for very small $f_{\mathrm{s}}$ and the adiabatic approximation fails. It was shown to be connected with the properties of systems with OOI which tend to average the influence of the periodic multiplicative forcing over time.

The results presented in this paper are closely related to the observation of SR in a system with Pomeau-Maneville type-III intermittency [13]. However, the system (1) has more in common with utilizing dynamical threshold-crossing detectors in SR than the systems with "conventional" intermittency, in which a time sequence of chaotic and periodic phases with approximately the same amplitude of oscillations is observed. Though a discrete-time map (1) is only a mathematical model, the results of this paper may serve as a starting point for experimental observation of SR in several physical systems exhibiting OOI. They include e.g. investigation of the influence of periodic forcing on the dynamics of synchronized chaotic oscillators [27], as it is known that the loss of synchronization occurs via OOI or attractor bubbling $[28,17,18]$; this problem may also have interesting implications for the recently developed methods of secure communication [29]. It is also worth noting that one of the first experimental observations of OOI took place in chaotic spin-wave dynamics [30,31]. It offers the possibility to observe $\mathrm{SR}$ in a system exhibiting OOI in similar conditions as in the above-mentioned experiment of Ref. [13]. We hope that these problems will be addressed in our future work.

\section{References}

[1] R. Benzi, A. Sutera, A. Vulpiani, J. Phys. A 14, L453 (1981).

[2] P. Jung, Phys. Rep. 234, 175 (1993).

[3] F. Moss, D. Pierson, D. O'Gorman, Int. J. Bifurcation Chaas 4, 1383 (1994).

[4] Proc. NATO Advanced Research Workshop: Stochastic Resonance in Physies and Biology, Eds. F. Moss, A. Bulsara, M.F. Shlesinger, J. Stat. Phys. 70, No. 1/2 (1993).

[5] Proc. Int. Workshop "Fluctuations in Physics and Biology: Stochastic Resonance, Signal Processing and Related Phenomena", Elba, 5-10 June 1994, Nuovo Cimento $D$ 17, No. 7-8 (1995).

[6] B. McNamara, K. Wiesenfeld, Phys. Rev. A 39, 4854 (1989).

[7] N.G. Stocks, N.D. Stein, P.V.E. McClintock, J. Phys. A 26, L385 (1993).

[8] K. Wiesenfeld, D. Pierson, E. Pantazelou, C. Dames, F. Moss, Phys. Rev. Lett. 72, 2125 (1994).

[9] Z. Gingl, L.B. Kiss, F. Moss, Europhys. Lett. 29, 191 (1995).

[10] G. Nicolis, C. Nicolis, D. McKernan, in Ref. [4], p. 125; V.S. Anischenko, A.B. Neiman, M.A. Safonova, in Ref. [4], p. 183. 
[11] V.S. Anischenko, M.A. Safonova, L.O. Chua, Int. J. Bifurcation Chaos 2, 397 (1992).

[12] V.S. Anischenko, M.A. Safonova, L.O. Chua, Int. J. Bifurcation Chaos 4, 441 (1994).

[13] E. Reibold, W. Just, J. Becker, H. Benner, Phys. Rev. Lett. 78, 3101 (1997).

[14] N. Platt, E.A. Spiegel, C. Tresser, Phys. Rev. Lett. 70, 279 (1993).

[15] J.F. Heagy, N. Platt, S.M. Hammel, Phys. Rev. E 49, 1140 (1994).

[16] N. Platt, S.M. Hammel, J.F. Heagy, Phys. Rev. Lett. 72, 3498 (1994).

[17] P. Ashwin, J. Buescu, I. Stewart, Phys. Lett. A 193, 126 (1994).

[18] S.C. Venkataramani, B.R. Hunt, E. Ott, Phys. Rev. E 54, 1346 (1996).

[19] S.C. Venkataramani, B.R. Hunt, E. Ott, D.J. Gauthier, J.C. Bienfang, Phys. Rev. Lett. 77, 5361 (1996).

[20] Ying-Cheng Lai, Phys. Rev. E 54, 321 (1996).

[21] E. Ott, J.C. Sommerer, Phys. Lett. A 188, 39 (1994).

[22] F. Chapeau-Blondeau, Phys. Rev. E 53, 5469 (1996).

[23] P. Jung, P. Hänggi, Phys. Rev. A 44, 8032 (1991).

[24] R. Bartussek, P. Hänggi, P. Jung, Phys. Rev. E 49, 3930 (1994).

[25] P. Jung, G. Mayer-Kress, in Ref. [5], p. 827.

[26] K. Wiesenfeld, K. DeMino, in Ref. [5], p. 977.

[27] L.M. Peccora, T.J. Carroll, Phys. Rev. Lett. 64, 821 (1990).

[28] A. Čenys, A. Namajūnas, A. Tamaševičius, T. Schneider, Phys. Lett. A 213, 259 (1996).

[29] K.M. Cuomo, A.V. Oppenheim, Phys. Rev. Lett. 71, 65 (1993).

[30] F. Rödelsperger, A. Cenys, H. Benner, Phys. Rev. Lett. 75, 2594 (1995).

[31] A. Krawiecki, A. Sukiennicki, Acta Phys. Pol. A 88, 269 (1995). 